\title{
Comment on "Earthquake cycles and physical modeling of the process leading up to a large earthquake"
}

\author{
A. Bizzarri ${ }^{1}$ and M. Cocco $^{2}$ \\ ${ }^{1}$ Istituto Nazionale di Geofisica e Vulcanologia, Sezione di Bologna, Bologna, Italy \\ ${ }^{2}$ Istituto Nazionale di Geofisica e Vulcanologia, Sezione di Sismologia e Tettonofisica, Roma, Italy
}

(Received March 11, 2005; Revised July 21, 2005; Accepted July 22, 2005; Online published December 25, 2006)

The modeling of earthquake initiation and subsequent rupture propagation requires the use of a fault constitutive law controlling the traction evolution and the slip acceleration that yields a finite energy flux at the crack tip. The determination of the temporal evolution of dynamic traction during the propagation of an earthquake rupture has been the major task of many recent investigations. Ohnaka (2004) presented a detailed discussion in favor of a " $r a$ tional" governing law for earthquake ruptures which is consistent with laboratory experiments and, according to the author, is based on the "physics of rock friction and fracture". He concluded that this constitutive law must be slip-dependent because the slip dependency is a "more fundamental property of the shear rupture than the ratedependency". Ohnaka (2004) also suggested that this slipdependent law model the whole seismic cycle (therefore including earthquake nucleation and long-term fault restrengthening) and considered the proposed law to be a governing relation unifying the different features of the earthquake failure process.

In order to support the proposed slip-dependent law, Ohnaka (2004) presented a questionable comparison between the slip- and the rate- and state-dependent (referred to as RS hereafter) constitutive laws (see section 2 of that paper). As previously mentioned, his discussion focused on demonstrating that the slip dependency is more important than the rate dependency. The goal of the present paper is not to open a debate on the more appropriate constitutive law, neither to support RS friction in favor of slip-dependent laws. Instead, our aim is to provide an alternative interpretation of the slip dependency of shear traction as well as to discuss the reasons why RS friction laws yield a traction evolution consistent with slip-weakening (referred to as SW hereafter). Although we share a common goal with Ohnaka in our search for the best framework within which to define a unified constitutive law that models earthquake initiation, propagation and arrest, we think that the complexity and the diversity of real fault zone structures make this task difficult to accomplish. Our reasoning is explained in detail in the following discussion.

Copyright (c) The Society of Geomagnetism and Earth, Planetary and Space Sciences (SGEPSS); The Seismological Society of Japan; The Volcanological Society of Japan; The Geodetic Society of Japan; The Japanese Society for Planetary Sciences; TERRAPUB
It is generally accepted that the dynamic fault weakening that occurs during the rupture propagation can be represented by a traction evolution showing a dominant SW behavior (Fig. 1). This evolution is characterized by a traction increase to the upper yield stress $\left(\tau_{u}\right)$, which is followed by a decrease to a kinetic friction level $\left(\tau_{f}\right)$ during a time interval that defines the duration of the breakdown process. The amount of slip required to the traction drop from the peak stress to the kinetic friction level is named the characteristic slip-weakening distance $\left(d_{0}\right)$. The cohesive zone represents the region of shear stress degradation near the tip of a propagating rupture (Ida, 1972; Andrews, 1976a, 1976b; Ohnaka and Yamashita, 1989). Slip-weakening has been observed in laboratory experiments (Okubo and Dieterich, 1984; Ohnaka et al., 1987), it has been proposed in theoretical studies (Campillo and Ionescu, 1997; Ohnaka, 2003 and references therein) and it has been used in numerical simulations of earthquake ruptures (Day, 1982; Olsen et al., 1997, among many others). The SW behavior of shear traction evolution has been demonstrated to also occur when RS friction laws are adopted (see Bizzarri and Cocco, 2003 and references therein). Therefore, a generally accepted consensus exists among researchers that SW characterizes dynamic fault weakening. It is also accepted that the shape of the traction versus slip curve can be nonlinear (i.e. $\partial \tau / \partial u \neq$ constant, where $\tau$ is the shear traction and $u$ is the fault slip; see Abercrombie and Rice, 2005) and may exhibit a slip-hardening phase that precedes the slip-weakening phase (i.e. $\tau$ increases with $u$ and reaches its peak value in the correspondence of a fault slip value $D_{a}$; see, for example, Ohnaka and Yamashita, 1989). For this reason, in many numerical simulations of dynamic rupture propagation, a SW constitutive law (Andrews, 1976a, 1976 b) is assumed in order to prescribe the traction evolution within the cohesive zone and to model the propagation of seismic waves in the medium surrounding the fault. However, many questions still remain unanswered with respect to the physical processes affecting the dynamic traction evolution and therefore controlling SW behavior. Also associated to this issue is the problem of choosing the best analytical formulation to appropriately model the constitutive behavior as well as to decide the independent physical observables adopted in the proposed law (see, for example, equation (2) in Bizzarri and Cocco, 2005). 


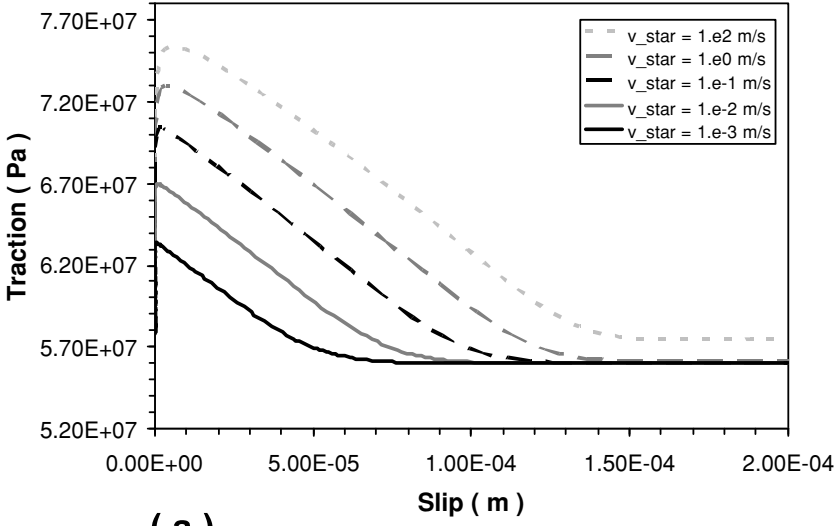

(a)

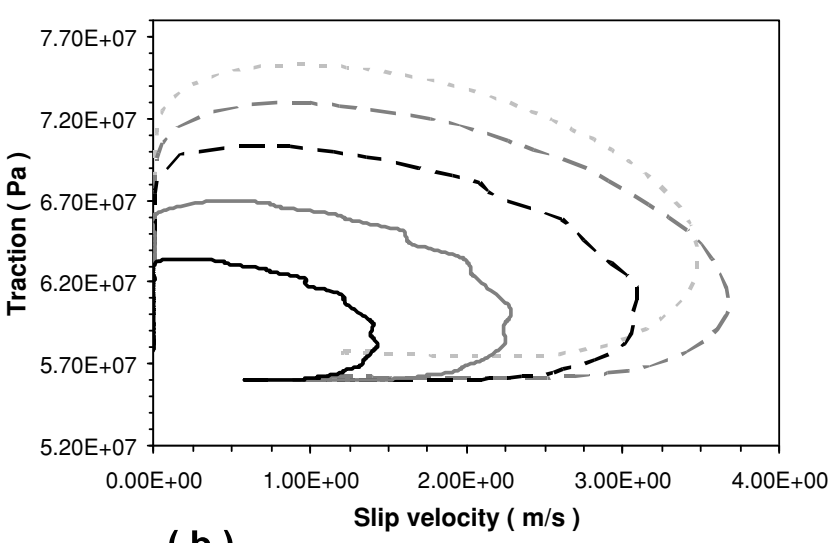

(b)

Fig. 1. Results of numerical experiments of a 2-D pure in-plane fully dynamic spontaneous rupture on a planar fault obeying to Eq. (1). The numerical method is the Finite Difference code described in Bizzarri et al. (2001), and the constitutive parameters are those cited in the caption of figure 1 of Cocco and Bizzarri (2002). Different simulations are performed changing only the value of the reference fault slip velocity $v_{*}$ from $1 \mathrm{~mm} / \mathrm{s}$ up to $100 \mathrm{~m} / \mathrm{s}$ and, consequently, the value of the initial pre-stress, which is assumed to be the steady state (see Bizzarri et al., 2001 for further numerical details). (a) Traction vs. slip (i.e., slip-weakening curve); (b) Traction vs. slip velocity (i.e., phase portrait). The two solid lines indicate the curves corresponding to laboratory values, while dashed curves are computed for larger values of $v_{*}$.

Ohnaka (2004) expressed serious concerns and criticism on the use of the RS friction laws as made by Bizzarri et al. (2001) and Cocco and Bizzarri (2002), considering one of the conclusions of these authors - that in order to have SW with RS-dependent friction laws, there is no need to assume that friction is independent of slip rate-to be "logically inconsistent". We believe that there are several shortcomings in Ohnaka's discussion of this issue; as such, our aim here is to provide a better explanation of the manner in which RS friction laws can yield a traction evolution showing SW during the spontaneous rupture propagation. To this end, we first remind the constitutive relations characterizing the slowness law used by Cocco and Bizzarri (2002) and Bizzarri and Cocco (2003), which is defined by two coupled equations:

$$
\begin{aligned}
\tau & =\left[\mu_{*}-a \ln \left(\frac{v_{*}}{v}+1\right)+b \ln \left(\frac{\Psi v_{*}}{L}+1\right)\right] \sigma_{n}^{\text {eff }} \\
\frac{d}{d t} \Psi & =1-\frac{\Psi v}{L}
\end{aligned}
$$

The former is often named the governing relation, while the latter is called the evolution equation. In Eq. (1), $v$ is the slip velocity, $\Psi$ is the state variable, $\mu_{*}$ and $v_{*}$ are reference values for the friction coefficient and for the slip velocity, respectively, $a, b$ and $L$ are the three constitutive parameters and $\sigma_{n}^{\text {eff }}$ is the effective normal stress. Dieterich and Kilgore (1996) pointed out that, if the slip velocity is constant and $\Psi v / L \gg 1$, the state variable evolution can be written as $\Psi=\Psi_{0} \exp (-u / L)\left(\Psi_{0}\right.$ being the initial value of the state variable) and, therefore, the slip dependence of total traction can be derived analytically. However, the assumption of a constant slip rate is not applicable to the dynamic traction evolution within the cohesive zone: it is well known that slip velocity varies over several orders of magnitude during the breakdown time (see Fig. 1). Using the results of their numerical simulations, Cocco and Bizzarri (2002) were able to demonstrate that RS-dependent friction laws yield a dynamic traction evolution characterized by SW, with slip velocity not being constant and vary- ing up to several meters per second. Thus, they concluded that the assumption of a constant slip velocity is not necessary to have a SW behavior of dynamic traction. We believe that this conclusion is correct. Ohnaka (2004) considers this issue "logically inconsistent" because of the effect of the high-velocity cutoff incorporated into Eq. (1) resulting from the presence of the +1 term in the arguments of the logarithms. To counter Ohnaka's arguments, it first has to be emphasized that, even if the first logarithmic term in Eq. (1) would saturate at high slip rates, the traction dependence on slip rate would remain in the governing equation because the state variable also depends on the fault slip velocity. Therefore, we stress the point that the slip velocity dependence of the shear traction is contained in both logarithmic terms and not only in the first one (usually named the "direct effect of friction"). This is clearly demonstrated by the simulations presented in Tinti et al. (2004) who have shown that, even using a different high slip velocity cutoff for the direct effect of friction, the SW behavior is always retrieved. Moreover, we have verified that the effect of the +1 terms does not affect the traction evolution when $v_{*}$ is greater than $0.1 \mathrm{~m} / \mathrm{s}$ (this condition was adopted in the simulations shown by Cocco and Bizzarri, 2002). For values of $v_{*}$ smaller than $0.1 \mathrm{~m} / \mathrm{s}$, the +1 term does affect the direct effect of friction; however, also in this case the fault shear traction still displays an evident SW behavior driven by the state variable evolution (see figure 1 and Bizzarri and Cocco, 2003, for details).

We agree with Ohnaka (2004) that the $v_{*}$ term cannot have an arbitrary value and has to be constrained by observations. In the literature, the only direct experimental measure of parameter $v_{*}$ is reported by Okubo and Dieterich (1986), who performed laboratory experiments and interpreted their observations using the RS formulation. They estimated (see table 1 in Okubo and Dieterich, 1986) the parameter $c_{3}$, which is equivalent to $v_{*} / L$ in our notation. It is important to note that, according to these experimental results, the value of $v_{*}$ depends on the scale at which the numerical experiment is performed: at the laboratory scale 
(fault size of the order of meters and $L \sim 1 \mu \mathrm{m}$ ), $v_{*} \sim 0.0001$ $\mathrm{m} / \mathrm{s}$, while for a real-world fault (size of the order of kilometers and $L \sim 1 \mathrm{~cm}$; Scholz, 1998), $v_{*} \sim 0.15 \mathrm{~m} / \mathrm{s}$. Bizzarri and Cocco (2003) demonstrated that the scaling between $d_{0}^{\text {eq }}$ and $L$ depends on the initial configuration of the sliding surface. This is also consistent with Ohnaka's $(2003,2004)$ conclusions that the characteristic SW distance and fracture energy are scale-dependent quantities. Therefore, we believe that the simulations presented by Cocco and Bizzarri (2002) and Bizzarri and Cocco (2003) demonstrate that RS friction laws yield a traction evolution within the cohesive zone consistent with the SW behavior. To this end we wish to emphasize the similarity between the traction evolution with slip inferred in numerical simulations governed by RSdependent friction laws (see Fig. 1) and the experimental results displayed by Ohnaka and Yamashita (1989) in figure 2 of their paper. In our opinion, this similarity represents a further validation of the agreement between numerical simulations and observational evidence.

On the basis of the results of laboratory and numerical experiments, we agree with Ohnaka that the traction evolution within the cohesive zone during the dynamic rupture propagation must show a SW behavior. Nevertheless, we disagree with his statement that this is the "unified" constitutive law that must be slip-dependent. Different constitutive relations allow the simulation of a traction evolution consistent with SW, each having different independent physical observables to analytically represent shear traction. We have discussed slip-dependent and RS-dependent friction laws, but other mechanisms can also affect the constitutive process and the dynamic traction evolution that might be described by different physical parameters (see Sleep, 1997). In other words, we believe that there are many competing physical mechanisms (such as mechanical lubrication or thermal pressurization) controlling the constitutive process that are not necessarily described by a slip-dependent law and that can yield a dynamic traction evolution characterized by SW. These distinct physical mechanisms can be adopted to describe tectonic faults because of the diversity of real fault zones.

Recent observations of active faults suggest that the fault zone consists of a damage zone containing a narrow fault core where a relatively thin slipping zone $(\mathrm{mm} / \mathrm{cm}$ thickness) is located (Chester and Chester, 1998; Sibson, 2003 and references therein). In that region, different physical mechanisms may occur: intact rock fracturing, comminution and pulverization (Wilson et al., 2004), subsequent gouge creation, gouge fragmentation (Sleep et al., 2000), frictional heating and melting as well as chemical environment changes. Moreover, the seismogenic zone may contain fluids that can migrate and affect the fault weakening, through hydrodynamic lubrication (Brodsky and Kanamori, 2001) and thermal pressurization (Andrews, 2002; Bizzarri and Cocco, 2004, 2006a, 2006b). We would also emphasize that the behavior of the damage zone (anelastic deformations, permeability and porosity evolutions) may influence the fault friction. All of these phenomena should be considered in the formulation of a unifying constitutive model that describes the evolution of the slipping surface. A likely expectation is that different configurations of fault zones will be described by different constitutive relations, and this has indeed been proposed in the literature. For example, the evolution of gouge materials has been simulated in the laboratory (Mair and Marone, 2000; Richardson and Marone, 1999) and in numerical experiments (Sleep et al., 2000). Therefore, within this diversity of possible configurations of a fault zone, we do not believe that it is justified to state that the constitutive law representing these processes must be slip-dependent and that this slip dependency is associated to the "correct representation of the physics of friction and fracture".

Recent results from laboratory experiments further corroborate that a slip-dependent constitutive law cannot account for the distinct physical processes characterizing faulting and dynamic rupture episodes during earthquakes. Di Toro et al. (2004) have shown an extraordinary progressive decrease in frictional resistance with increasing slip velocity: these experimental results suggest that the friction coefficient (that is, at constant normal stress, the shear traction) depends both on slip and slip velocity. Tullis and Goldsby (2003) have modeled these data and interpreted the friction reduction at high slip rates in terms of flash heating of contact asperities (Rice, 1999). Mair and Marone (2000) performed laboratory experiments to study the friction of simulated fault gouge at relatively high normal stress (25-70 MPa) and found a dramatic reduction in frictional strength with accumulated slip. They interpreted their laboratory results in terms of a slip-dependent textural evolution of gouge and found an evident velocity-weakening behavior as well as negligible shear heating. Thus, many observations and laboratory experiments suggest that fault gouge and the properties of contact surface evolve during slip episodes; this evolution depends on the slip, slip rate, normal stress as well as the degree of strain localization. Our opinion is that the interpretation of these observations does not allow us to distinguish the independent variables controlling the dynamic fault weakening.

It is important to point out that the different physical mechanisms described above represent either microscopic or macroscopic processes. Melting caused by frictional heating at high slip rates (Tsutsumi and Shimamoto, 1997) represents a further macroscopic frictional behavior of a fault zone (see also Brodsky and Kanamori, 2001). Ohnaka (2003) emphasizes that the shear traction and the slip appearing in the constitutive formulation have to be regarded as macroscopic quantities (i.e., defined as macroscopic averages) describing the shear stress acting on both walls of the fault zone thickness as well as their relative displacement. In this context, the shear stress adopted in the constitutive law and used to characterize the macroscopic dynamic fault weakening is not the shear stress acting on an individual asperity of contact surface, micro-crack or gouge fragment within the slipping zone. We fully agree with this view of the earthquake failure process: given our present state of knowledge we cannot expect to be able to model the details and the complexity of the inhomogeneous processes occurring in the localized zone where the strain is concentrated. For the same reasons we believe that a simple slip-dependent law cannot be proposed to characterize the traction evolution. Further investigations are needed to 
answer this problem quantitatively.

As the next logical step to this discussion, we would like to propose an alternative interpretation of the shear traction dependence on slip (i.e., the SW behavior). Our opinion is that if we restrict our goal to the search for an analytical expression capable of representing the traction evolution within the cohesive zone during the dynamic earthquake propagation, then a slip-dependent law is a candidate solution. In this case, a "unifying" slip-dependent law means that we only reproduce the traction evolution independently of the physical mechanisms controlling the constitutive process. In this context, we agree with the Ohnaka's conclusions. Conversely, we believe that the goal of representing the whole variety of constitutive processes and the dynamic fault weakening during earthquake failures with a slip-dependent law might be misleading for future developments of research in this field.

Acknowledgments. We would thank Eiichi Fukuyama, Raul Madariaga and an anonymous reviewer for useful discussions.

\section{References}

Abercrombie, R. E. and J. R. Rice, Can observations of earthquake scaling constrain slip weakening?, Geophys. J. Int., 162, 406-424, 2005.

Andrews, D. J., Rupture propagation with finite stress in antiplane strain, J. Geophys. Res., 81, 3575-3582, 1976 a.

Andrews, D. J., Rupture velocity of plane strain shear crack, J. Geophys. Res., 81, 5679-5687, 1976b.

Andrews, D. J., A fault constitutive relation accounting for thermal pressurization of pore fluid, J. Geophys. Res., 107(B12), 2363, doi: 10.1029/ 2002JB001942, ESE 15-1-15-8, 2002.

Bizzarri, A. and M. Cocco, Slip-weakening behavior during the propagation of dynamic ruptures obeying rate- and state-dependent friction laws, J. Geophys. Res., 108(B8), 2373, doi: 10.1029/2002JB002198, 2003.

Bizzarri, A. and M. Cocco, Thermal pressurization in 3-D dynamic spontaneous rupture models with cohesive zone, Eos Trans. AGU, 85(47), Fall Meet. Suppl., Abstract T23A-0572, 2004.

Bizzarri, A. and M. Cocco, 3D dynamic simulations of spontaneous rupture propagation governed by different constitutive laws with rake rotation allowed, Ann. Geophys., 48(2), 279-299, 2005.

Bizzarri, A. and M. Cocco, A thermal pressurization model for the spontaneous dynamic rupture propagation on a three-dimensional fault: 1. Methodological approach, J. Geophys. Res., 111, B05303, doi:10.1029/2005JB003862, 2006a

Bizzarri, A. and M. Cocco, A thermal pressurization model for the spontaneous dynamic rupture propagation on a three-dimensional fault: 2 . Traction evolution and dynamic parameters, J. Geophys. Res., 111, B05304, doi:10.1029/2005JB003864, 2006b.

Bizzarri, A., M. Cocco, D. J. Andrews, and E. Boschi, Solving the dynamic rupture problem with different numerical approaches and constitutive laws, Geophys. J. Int., 144, 656-678, 2001.

Brodsky, E. E. and H. Kanamori, Elastohydrodynamic lubrication of faults, J. Geophys. Res., 106(B8), 16,357-16,374, 2001

Campillo, M. and I. R. Ionescu, Initiation of antiplane shear instability under slip dependent friction, J. Geophys. Res., 102(B9), 20,363-20,371, 1997.

Chester, F. M. and J. S. Chester, Ultracataclasite structure and friction processes of the Punchbowl fault, San Andreas system, California, Tectonophysics, 295, 199-221, 1998.

Cocco, M. and A. Bizzarri, On the slip-weakening behavior of rate- and state-dependent constitutive laws, Geophys. Res. Lett., 29(11), 11-1-114, 2002.

Day, S. M., Three-dimensional finite difference simulation of fault dynamics: Rectangular faults with fixed rupture velocity, Bull. Seismol. Soc. Am., 72, 705-727, 1982.

Di Toro, G., D. L. Golsdby, and T. T. Tullis, Friction falls towards zero in quartz rock as slip velocity approaches seismic rates, Nature, $\mathbf{4 2 7}$, 436-439, 2004.

Dieterich, J. H. and B. Kilgore, Implications of fault constitutive properties for earthquake prediction, Proc. Natl. Acad. Sci. USA, 93, 3787-3794, 1996.

Ida, Y., Cohesive force across the tip of a longitudinal-shear crack and Griffith's specific surface energy, J. Geophys. Res., 77(20), 3796-3805, 1972.

Mair, K. and C. Marone, Shear heating in granular layers, Pure Appl. Geophys., 157, 1847-1866, 2000.

Ohnaka, M., A constitutive scaling law and a unified comprehension for frictional slip failure, shear fracture of intact rock, and earthquake rupture, J. Geophys. Res., 108, 2080, doi: 10.1029/2002JB000123, 2003.

Ohnaka, M., Earthquake cycles and physical modeling of the process leading up to a large earthquake, Earth Planets Space, 56, 773-793, 2004.

Ohnaka, M. and T. Yamashita, A cohesive zone model for dynamic shear faulting based on experimentally inferred constitutive relation and strong motion source parameters, J. Geophys. Res., 94(B4), 4089-4104, 1989.

Ohnaka, M., Y. Kuwahara, and K. Yamamoto, Constitutive relations between dynamic physical parameters near a tip of the propagation slip zone during stick-slip shear failure, Tectonophysics, 144, 109-125, 1987.

Okubo, P. G. and J. H. Dieterich, Effects of physical fault properties on frictional instabilities produced on simulated faults, J. Geophys. Res., 89, 5817-5827, 1984 .

Okubo, P. G. and J. H. Dieterich, State variable fault constitutive relations for dynamic slip, in Earthquake Source Mechanics, Geophysical Monograph, 37, Maurice Ewing Series, 6, edited by S. Das, J. Boatwright, and C. Scholz, Am. Geophys. Union, Washington D.C., 25-35, 1986.

Olsen, K. B., R. Madariaga, and R. J. Archuleta, Three-dimensional dynamic simulation of the 1992 Landers earthquake, Science, 278, 834 838, 1997.

Rice, J. R., Flash heating at asperity contacts and rate-dependent friction, Eos Trans. AGU, 80(46), Fall Meet. Suppl.,p. F471, 1999.

Richardson, E. and C. Marone, Effects of normal stress vibrations on frictional healing, J. Geophys. Res., 104(B12), 28,859-28,878, 1999.

Scholz, C. H., Earthquakes and friction laws, Nature, 391, 37-42, 1998.

Sibson, R. H., Thickness of the seismic slip zone, Bull. Seism. Soc. Am., 93(3), 1169-1178, 2003.

Sleep, N. H., Application of a unified rate and state friction theory to the mechanics of fault zones with strain localization, J. Geophys. Res., 102(B2), 2875-2895, 1997.

Sleep, N. H., E. Richardson, and C. Marone, Physics of friction and strain rate localization in synthetic fault gouge, J. Geophys. Res., 106(B11), 25,875-25,890, 2000.

Tinti, E., A. Bizzarri, A. Piatanesi, and M. Cocco, Estimates of slip weakening distance for different dynamic rupture models, Geophys. Res. Lett., 31(L02611), doi: 10.1029/2003GL018811, 2004.

Tsutsumi, A. and T. Shimamoto, High-velocity frictional properties of gabbro, Geophys. Res. Lett., 24, 699-702, 1997.

Tullis, T. E. and D. Goldsby, Laboratory experiments on fault shear resistance relevant to coseismic earthquake slip, SCEC Annual Report for 2003, 2003.

Wilson, B., T. Dewers, and Z. Reches, Surface area and surface energy of fault gouge: observations of the San Andreas gouge in Tejon Pass area, California (abstract), Seismol. Soc. Am. Meet. 2004, 2004.

A. Bizzarri (e-mail: bizzarri@bo.ingv.it) and M. Cocco 